\title{
Lodos de esgoto alcalinizados em solos do estado do Paraná: taxa de aplicação máxima anual e comparação entre métodos para recomendação agrícola
}

\section{Alkaline sewage sludge on soils of Paraná state: maximum annual application rate and comparison between methods for agricultural recommendation}

\author{
Giovana Clarice Poggere \\ Engenheira Agrônoma pela Universidade Federal do Paraná (UFPR). Mestranda do Programa de Pós-graduação em Ciência do Solo da UFPR - Curitiba \\ (PR), Brasil.
}

\section{Beatriz Monte Serrat}

Engenheira Agrônoma pela UFPR. Doutora em Solos e Nutrição de Plantas pela Universidade de São Paulo (USP) - São Paulo (SP), Brasil. Professora do Programa de Pós-graduação em Ciência do Solo da UFPR - Curitiba (PR), Brasil.

\section{Antônio Carlos Vargas Motta}

Engenheiro Agrônomo pela Universidade Federal do Espírito Santo (UFES) - Vitória (ES), Brasil. Doutor em Solos e Agronomia pela Auburn University Auburn (AL), Estados Unidos da América. Professor do Programa de Pós-graduação em Ciência do Solo da UFPR - Curitiba (PR), Brasil.

\section{Simone Bittencourt}

Engenheira Agrônoma pela UFPR. Doutoranda do Programa de Pós-graduação em Engenharia de Recursos Hídricos e Ambiental da UFPR. Engenheira Agrônoma da Companhia de Saneamento do Paraná (SANEPAR) - Curitiba (PR), Brasil.

\author{
Maristela Dalpisol \\ Engenheira Agrônoma pela UFPR. Mestranda do Programa de Pós-graduação em Ciência do Solo da UFPR - Curitiba (PR), Brasil.
}

\section{Cleverson Vitório Andreoli}

Engenheiro Agrônomo pela UFPR. Doutor em Meio Ambiente e Desenvolvimento pela UFPR. Professor do Programa de Pós-graduação em Organizações e Sustentabilidade do Centro Universitário Franciscano (FAE) - Curitiba (PR) Brasil.

\section{Resumo}

Para o uso agrícola do lodo de esgoto higienizado por processo de estabilização alcalina prolongada (lodo EAP), além de estabelecer limites de aplicação máxima conforme a legislação, é necessário estudar atributos dos lodos e dos solos que favoreçam as metodologias de sua recomendação. Objetivou-se determinar a taxa de aplicação máxima anual (TAMA) de lodo EAP pelo critério de elevação do pH para 20 lodos e solos do estado do Paraná; determinar a influência dos atributos desses solos nos valores da TAMA de lodo EAP; avaliar o uso dos métodos de saturação por bases (V\%) e pH referência (SMP), utilizando como padrão o método da incubação. Empregou-se o ensaio de incubação para lodo EAP aplicado ao solo, conforme metodologia oficial. Os resultados indicam que as TAMA de lodo EAP variaram de $10 \mathrm{a}>80 \mathrm{Mg}^{-h^{-1}}$ para as 20 regionais estudadas; Os atributos químicos (capacidade de troca de cátions, $\mathrm{H}+\mathrm{Al}$ e carbono) e físico (argila) são os que melhor definem a determinação da TAMA, apresentando relação diretamente proporcional. Os métodos do $\mathrm{V} \%$ e do $\mathrm{pH}$ referência (SMP) para $\mathrm{pH}_{\mathrm{H}_{2} \mathrm{O}} 5,5$ mostraram-se seguros quanto à recomendação agrícola.

Palavas-chave: calagem; saturação por bases; resíduo orgânico alcalino. 


\section{Abstract}

For agricultural use of alkalinized sludge (AS), beyond to establish the maximum application limits to comply with the legislation, it is necessary to study the attributes of sludges and soils that favor the methodologies of its recommendation. The objectives of this study were to determine the maximum annual application rate (MAAR) of alkalinized sludge (AS) according to the pH elevation criterion for 20 sludge origins and soils of the Paraná state; to determine the influence of soil attributes over the MAAR of AS; to evaluate two methods rate calculation for AS, the base saturation (V\%) and pH reference (SMP) by comparing with the standard incubation methodology for AS sludge application. It was used incubation test for AS applied to soil, following official methodology. The results indicated that the MAAR were ranged 10 to $>80 \mathrm{Mg}$.ha ${ }^{-1}$, for the 20 regions; the chemical properties (cation exchange capacity, $\mathrm{H}+\mathrm{Al}$ and carbon) and physical properties (clay) were the best parameters to the determination of the MAAR, showing direct relationship; the V\% and SMP ( $\mathrm{pH}_{\mathrm{H}_{2} \mathrm{O}} 5$,5) methods were safe to the agricultural recommendation.

Keywords: liming; base saturation; organic waste alkali.

\section{Introdução}

Para o uso agrícola de lodos gerados em estações de tratamento de esgoto sanitário, a legislação federal define critérios e procedimentos, enquanto, a legislação estadual no Paraná a complementa, dispondo sobre licenciamento ambiental, estabelecendo condições e padrões ambientais, além de outras providências, para empreendimentos de saneamento.

Com base nessas legislações, a Companhia de Saneamento do Paraná (SANEPAR) desenvolve o Programa de Utilização Agrícola de Lodo de Esgoto, visando proporcionar a disposição final adequada, melhoria das condições dos solos e aumento de produtividade das culturas agrícolas (BITTENCOURT et al., 2009).

As Estações de Tratamento de Esgoto (ETE) operacionalizadas pela SANEPAR utilizam o sistema de digestão anaeróbia e, segundo Pike e Davis (1984), neste tratamento, a eficácia na redução dos patógenos depende do tempo e da temperatura, sendo em média de 60 a $75 \%$ em processos com temperatura de $20^{\circ} \mathrm{C}$ por 30 dias. Portanto, para a completa redução dos patógenos, são necessários tratamentos de higienização complementares.

Nessas ETE, a higienização dos lotes de lodo é feita pelo processo de Estabilização Alcalina Prolongada (EAP), que tem por princípio a elevação do $\mathrm{pH}$ do lodo a níveis iguais ou superiores a 12 , promovido pela adição de material alcalino, que após a cura por 30 dias, inativa ou destrói a maior parte dos patógenos (PARANÁ, 2009).

Os lotes de lodo higienizados nas ETE das Unidades de Gerenciamento de Lodo (UGL) do Paraná são monitorados para a determinação do potencial agronômico, através de análises de substâncias orgânicas, sanidade, metais pesados e estabilidade. Esse monitoramento permite a realização de ações que visam garantir que o material disponibilizado aos agricultores apresente a qualidade exigida pelas normas da legislação vigente (BRASIL, 2006; PARANÁ, 2009). Embora promova aumento no volume final do resíduo, o método EAP apresenta-se viável, pois gera um material sanitariamente adequado que além de atuar como fonte de nutrientes (CHUEIRI et al., 2007; CORREAA; WHITE; WEATHERLEY, 2005), promove aumento no pH do solo (FIA; MATOS; AGUIRRE, 2005).
Andreoli, Pegorini e Fernandes (2001), baseados no método de saturação por bases (V\%), o qual sistematiza alguns atributos de fertilidade do solo, consideraram o poder de neutralização (PN) da cal adicionada ao lodo bruto na determinação da quantidade de lodo EAP a ser aplicada em cada área agrícola. Contudo, a partir da Resolução CONAMA no 375/06 (BRASIL, 2006), a recomendação do uso do lodo de esgoto alcalinizado, além dos demais critérios nela citados, deve levar em conta os resultados dos ensaios de elevação de $\mathrm{pH}$ provocados por este resíduo no solo predominante da região, de modo a garantir que o $\mathrm{pH}$ final da mistura solo-lodo de esgoto não ultrapasse o limite de sete. Devido à sua precisão, esse método da incubação tem sido utilizado como método padrão para avaliar o efeito corretivo de diversos materiais (MOTTA \& LIMA, 2006; NOLLA \& ANGHINONI, 2004), porém sua execução é morosa.

Marin et al. (2010) avaliaram curvas de incubação com lodo EAP em diferentes classes de solo da região metropolitana de Curitiba (RMC) e observaram valores de taxas variáveis, sendo o maior valor (49 Mg.ha-1) obtido para o solo que apresentava $\mathrm{pH}_{\mathrm{CaCl}_{2}}$ baixo e valores altos de acidez potencial, carbono e Al trocável. Serrat et al. (2011), trabalhando com dois solos da RMC incubados com lodo EAP da ETE São Jorge, Almirante Tamandaré (PR), verificaram interação entre o lodo EAP e o tipo de solo, sendo que a taxa máxima no Latossolo Bruno, que apresentava maior poder tampão, foi de $74 \mathrm{Mg} \mathrm{ha}^{-1}$, 1,8 vezes mais que no Cambissolo Háplico. Quando os mesmos autores avaliaram, no Cambissolo a reação de dois lodos, um misto coletado na UGL e higienizado em escala real (PN=51,1\%) e outro em uma das ETE e higienizado em laboratório ( $\mathrm{PN}=29,4 \%)$, observaram que a taxa máxima sofreu influência dos lodos, sendo maior no que apresentava menor PN.

Assim, estudos a respeito dos aspectos específicos relacionados à reação dos lodos EAP em solos com diferentes características devem ser aprofundados, uma vez que as variações, tanto das características dos solos quanto dos lodos de cada região, podem influenciar na taxa de aplicação máxima anual deste material.

Desta forma, objetivou-se com esse trabalho, determinar a taxa de aplicação máxima anual (TAMA) de lodo EAP pelo critério 
de elevação do pH do solo para diversas regiões do estado do Paraná; determinar os atributos do solo que mais influenciam nos valores da taxa de aplicação máxima anual de lodo EAP; avaliar os métodos de saturação por bases (V\%) e pH referência (SMP) para recomendação agrícola de corretivos, utilizando como padrão o método da incubação.

\section{Metodologia}

Para cada uma das 20 regionais da SANEPAR, selecionou-se uma Estação de Tratamento de Esgoto (ETE) com lodo gerado por tratamento anaeróbio, onde se coletou lodo recentemente desaguado em leito de secagem. Nessa amostra, foram determinados o pH e, após secagem em estufa até peso constante, o teor de sólidos totais (ST). A higienização do lodo foi feita pelo processo de Estabilização Alcalina Prolongada (EAP) onde, a partir do valor de ST do lodo seco, adicionou-se a quantidade de cal no lodo úmido na proporção de 50\% dos ST e misturou-se de forma homogênea, permanecendo em cura por 30 dias. As análises do lodo bruto e higienizado foram realizadas pelo laboratório da Unidade de Gerenciamento de Lodo (UGL) CIC/Xisto. $O$ poder de neutralização (PN) da cal e dos lodos higienizados foi determinado segundo metodologia de Tedesco et al. (1995), visando a caracterização do potencial corretivo de cada lodo alcalinizado (Tabela 1).

Nas 20 regionais, selecionou-se também um tipo de solo que representasse os solos agrícolas da região, amostrados em área sem adição de calagem, pelo menos nos últimos 5 anos. Com auxílio do ponto de localização fornecido por aparelho de georeferenciamento global (GPS), obteve-se a classificação do solo de acordo com Bhering e Santos (2008) (Tabela 2).

As análises para caracterização física e química dos solos seguiram metodologia descrita por EMBRAPA (1997) e Marques e Motta (2003) (Tabelas 2 e 3), respectivamente. A classificação dos atributos químicos e físicos em níveis baixo, médio e alto, foi realizada conforme Serrat, Krieger e Motta (2006). A umidade na capacidade de

Tabela 1 - Origem e caracterização de lodos brutos e higienizados das 20 regionais do estado do Paraná e da cal utilizada no processo de higienização dos lodos.

\begin{tabular}{|c|c|c|c|c|c|c|c|}
\hline \multirow{3}{*}{ Regional } & \multirow{3}{*}{ ETE } & \multicolumn{6}{|c|}{ Lodo } \\
\hline & & \multicolumn{3}{|c|}{ Bruto desaguado } & \multicolumn{3}{|c|}{ Higienizado* } \\
\hline & & $\begin{array}{l}\text { ST } \\
(\%)\end{array}$ & $\mathrm{pH}$ & $\begin{array}{l}\text { PRL } \\
\text { (dias) }\end{array}$ & $\begin{array}{l}\text { ST } \\
(\%)\end{array}$ & $\mathrm{pH}$ & $\begin{array}{l}\mathrm{PN} \\
(\%)\end{array}$ \\
\hline Francisco Beltrão & Marrecas & 48,5 & 3,8 & 34 & 64,3 & 13,5 & 40 \\
\hline Cascavel & Norte & 26,1 & 8,4 & 45 & 76,4 & 9,5 & 46 \\
\hline Região Metropolitana de Curitiba & São Jorge & 36,4 & 6,8 & 54 & 50 & 11,6 & 30 \\
\hline Pato Branco & Ligeiro & 42,0 & 4,9 & 92 & 56,2 & 10,4 & 31 \\
\hline Guarapuava & Sul II & 52,8 & 6,9 & 17 & 64,7 & 13,2 & 54 \\
\hline Toledo & Santo Campagnolo & 26,7 & 5,4 & 21 & 71,4 & 9,3 & 40 \\
\hline Apucarana & Cambará & 19,3 & 7,1 & 13 & 29,9 & 13,0 & 33 \\
\hline Campo Mourão & Rio km 119 & 65,5 & 4,4 & 35 & 89,6 & 12,9 & 46 \\
\hline União da Vitória & Estação Nova & 54,4 & 5,5 & 59 & 78,3 & 12,9 & 47 \\
\hline Litoral & Matinhos & 26,2 & 6,8 & 27 & 84,2 & 12,2 & 52 \\
\hline Telêmaco Borba & Uvaranal & 49,0 & 5,8 & 38 & 57,4 & 13,0 & 45 \\
\hline Maringá & Mandacaru & 33,6 & 4,0 & 28 & 51,7 & 13,0 & 34 \\
\hline Santo Antônio da Platina & Boi Pintado & 56,1 & 2,9 & 92 & 71,3 & 13,5 & 44 \\
\hline Londrina & Norte & 28,0 & 4,4 & 48 & 45,9 & 12,8 & 39 \\
\hline Foz do Iguaçu & Ouro Verde & 71,0 & 2,4 & 60 & 81 & 12,6 & 46 \\
\hline Arapongas & Campinho & 43,8 & 7,8 & 14 & 55,4 & 13,0 & 41 \\
\hline Cornélio Procópio & Ribeirão dos Veados & 84,7 & 3,9 & 68 & 85,2 & 13,5 & 58 \\
\hline Paranavaí & Vila City & 23,0 & 7,9 & 27 & 34,7 & 13,0 & 42 \\
\hline Ponta Grossa" & Gertrudes & 53,0 & 5,5 & 23 & 48,8 & 13,1 & 59 \\
\hline Umuarama\# & Pinhalzinho & 83,9 & 5,7 & 19 & 65,4 & 13,8 & 40 \\
\hline Média & & 46,2 & 5,5 & 40,7 & 63,09 & 12,5 & 43 \\
\hline Material alcalinizante & & & $\mathrm{pH}$ & & PN & & PRNT \\
\hline Cal & & & 13,3 & & 124 & & 105,1 \\
\hline
\end{tabular}

ETE: Estação de Tratamento de Esgoto; ST: sólidos totais; PN: poder de neutralização; PRNT: poder relativo de neutralização total; PRL: período entre a retirada do lodo do reator/descarga no leito de secagem e a amostragem; *lodo higienizado pelo processo de estabilização alcalina prolongada, com cal a 50\% ST; \#lodos umedecidos durante a higienização. 
Tabela 2 - Classificação e atributos físicos dos 20 solos utilizados no processo de incubação, correspondente a cada regional atendida pela Companhia de Saneamento do Paraná.

\begin{tabular}{|c|c|c|c|c|c|c|c|}
\hline \multirow{2}{*}{ Regional da SANEPAR } & \multirow{2}{*}{$\begin{array}{l}\text { Local da coleta } \\
\text { (Município) }\end{array}$} & \multirow{2}{*}{ Solos } & Areia & Silte & Argila & \multirow{2}{*}{$\begin{array}{c}\text { Ds } \\
\left(\mathrm{g} \cdot \mathrm{cm}^{-3}\right)\end{array}$} & \multirow{2}{*}{$\underset{\left(\mathrm{g} \cdot \mathrm{kg}^{-1}\right)}{\mathrm{U}}$} \\
\hline & & & \multicolumn{3}{|c|}{$\left(\mathrm{g} \cdot \mathrm{kg}^{-1}\right)$} & & \\
\hline Francisco Beltrão & Francisco Beltrão & LVdf & 31,5 & 268,5 & 700,0 & 1,54 & 54,2 \\
\hline Cascavel & Cascavel & LVdf & 45,5 & 103,5 & 850,0 & 1,31 & 44,2 \\
\hline Região metropolitana de Curitiba & Pinhais & LBw & 221,0 & 129,0 & 650,0 & 0,95 & 54,3 \\
\hline Pato Branco & Pato Branco & LVdf & 19,0 & 131,0 & 850,0 & 0,95 & 61,2 \\
\hline Guarapuava & Guarapuava & LBd & 35,0 & 215,0 & 750,0 & 1,13 & 49,5 \\
\hline Toledo & Toledo & LVdf & 67,5 & 132,5 & 800,0 & 1,15 & 43,0 \\
\hline Apucarana & Apucarana & NVef & 58,5 & 191,5 & 750,0 & 0,96 & 48,9 \\
\hline Campo Mourão & Campo Mourão & LVdf & 70,5 & 129,5 & 800,0 & 1,13 & 44,8 \\
\hline União da Vitória & Rio Negro & RRdh & 446,5 & 328,5 & 225,0 & 1,31 & 40,0 \\
\hline Litoral & Matinhos & cXbd & 488,5 & 211,5 & 300,0 & 1,32 & 48,4 \\
\hline Telêmaco Borba & Telêmaco Borba & PVAd & 391,0 & 384,0 & 225,0 & 1,28 & 33,6 \\
\hline Maringá & Maringá I & NVef & 51,5 & 198,5 & 750,0 & 0,68 & 46,2 \\
\hline Santo Antônio da Platina & Santo Antônio da Platina & LVef & 300,5 & 274,5 & 425,0 & 1,66 & 19,8 \\
\hline Londrina & Londrina & LVd & 40,5 & 234,5 & 725,0 & 1,02 & 41,9 \\
\hline Foz do Iguaçu & Foz do Iguaçu & NVef & 24,0 & 326,0 & 650,0 & 1,42 & 36,8 \\
\hline Arapongas* & Maringá II & LVd & 701,0 & 49,0 & 250,0 & 1,02 & 29,0 \\
\hline Cornélio Procópio & Cornélio Procópio & NVef & 110,5 & 439,5 & 450,0 & 1,10 & 49,9 \\
\hline Paranavaí & Paranavaí & PVd & 803,5 & 47,5 & 150,0 & 1,35 & 13,2 \\
\hline Ponta Grossa & Ponta Grossa & LV & 646,5 & 78,5 & 275,0 & 1,49 & 24,3 \\
\hline Umuarama & Umuarama & LVd & 767,0 & 33,0 & 200,0 & 1,20 & 27,2 \\
\hline
\end{tabular}

SANEPAR: Companhia de Saneamento do Paraná; LVdf: Latossolo Vermelho distroférrico; LBw: Latossolo Bruno Ácrico; LBd: Latossolo Bruno distrófico; Nvef: Nitossolo Vermelho eutroférrico; RRdh: Neossolo Regolítico distro-húmbrico; CXbd: Cambissolo Háplico tb distrófico; PVAd: Argissolo Vermelho-Amarelo distrófico; LVd: Latossolo Vermelho distrófico; LV: Latossolo Vermelho (Bhering; Santos, 2008); Ds: densidade do solo; U: Umidade; *tipo de solo representativo da área correspondente à regional de Arapongas.

Tabela 3 - Atributos químicos dos 20 solos utilizados no processo de incubação, correspondente a cada regional atendida pela Companhia de Saneamento do Paraná.

\begin{tabular}{|c|c|c|c|c|c|c|c|c|c|c|c|}
\hline \multirow{3}{*}{$\begin{array}{l}\text { Local de coleta } \\
\text { (Município) } \\
\text { Francisco Beltrão }\end{array}$} & \multicolumn{2}{|c|}{$\mathrm{pH}$} & $\mathrm{Al}$ & \multirow[t]{2}{*}{$\mathrm{H}+\mathrm{Al}$} & \multirow{2}{*}{\multicolumn{2}{|c|}{$\begin{array}{l}\mathrm{Ca} \quad \mathrm{Mg} \\
\left(\mathrm{cmol}_{\mathrm{c}} \mathrm{dm}^{-3}\right)\end{array}$}} & \multirow[t]{2}{*}{$\mathrm{K}$} & \multirow[t]{2}{*}{ СТC } & \multirow{2}{*}{$\begin{array}{c}\mathrm{P} \\
\left(\mathrm{Mg} \cdot \mathrm{dm}^{-3}\right)\end{array}$} & \multirow{2}{*}{$\begin{array}{c}\mathrm{C} \\
\left(\mathrm{g} \cdot \mathrm{dm}^{-3}\right)\end{array}$} & \multirow{2}{*}{$\begin{array}{l}\text { V } \\
\%\end{array}$} \\
\hline & $\mathrm{CaCl}_{2}$ & SMP & & & & & & & & & \\
\hline & 4,1 & 4,3 & 5,3 & 17,6 & 2,5 & 1,4 & 0,44 & 21,94 & 3,6 & 19,2 & 20 \\
\hline Cascavel & 4,1 & 5,0 & 1,3 & 10,5 & 3,6 & 1,2 & 0,34 & 15,64 & 3,6 & 30,7 & 33 \\
\hline Pinhais* & 4,0 & 4,7 & 3,7 & 15,8 & 3,1 & 1,6 & 0,09 & 20,59 & 1,3 & 65,4 & 23 \\
\hline Pato Branco & 3,9 & 4,6 & 2,9 & 14,1 & 0,5 & 0,1 & 0,14 & 14,84 & 1,3 & 46,9 & 5 \\
\hline Guarapuava & 4,1 & 4,9 & 1,3 & 12,1 & 1,9 & 1,2 & 0,29 & 15,49 & 2,7 & 38,6 & 22 \\
\hline Toledo & 3,8 & 5,4 & 1,5 & 7,8 & 2,0 & 0,9 & 0,54 & 11,24 & 3,7 & 31,8 & 31 \\
\hline Apucarana & 4,9 & 5,9 & 0,1 & 5,4 & 4,2 & 2,3 & 0,50 & 12,40 & 2,2 & 24,3 & 56 \\
\hline Campo Mourão & 4,1 & 5,5 & 0,9 & 7,2 & 1,5 & 0,8 & 0,23 & 9,73 & 3,4 & 35,1 & 26 \\
\hline Rio Negro* & 3,8 & 5,2 & 2,7 & 9,0 & 0,2 & 0,2 & 0,14 & 9,54 & 5,9 & 30,7 & 6 \\
\hline Matinhos* & 3,7 & 5,4 & 2,3 & 7,8 & 0,1 & 0,2 & 0,08 & 8,18 & 3,2 & 28,5 & 5 \\
\hline Telêmaco Borba & 3,8 & 5,1 & 2,4 & 9,7 & 0,5 & 0,1 & 0,10 & 10,40 & 2,2 & 18,2 & 7 \\
\hline Maringá I & 4,4 & 5,6 & 0,3 & 6,7 & 4,8 & 1,8 & 0,29 & 13,59 & 3,6 & 45,7 & 51 \\
\hline Santo Antônio da Platina & 4,5 & 5,7 & 0,3 & 6,3 & 3,2 & 1,5 & 0,22 & 11,22 & 3,8 & 17,2 & 44 \\
\hline Londrina & 5,0 & 6,3 & 0,0 & 4,0 & 2,6 & 1,1 & 0,13 & 7,83 & 2,7 & 15,3 & 49 \\
\hline Foz do Iguaçu & 4,9 & 6,2 & 0,1 & 4,3 & 4,5 & 2,1 & 0,28 & 11,18 & 1,9 & 31,8 & 62 \\
\hline Maringá II* & 3,7 & 5,5 & 1,2 & 7,2 & 1,3 & 0,5 & 0,29 & 9,29 & 4,2 & 23,2 & 22 \\
\hline Cornélio Procópio & 4,7 & 5,4 & 0,2 & 7,9 & 4,0 & 1,4 & 0,58 & 11,38 & 3,8 & 25,3 & 53 \\
\hline Paranavaí & 4,5 & 6,9 & 0,2 & 2,5 & 0,7 & 0,4 & 0,07 & 3,67 & 1,4 & 5,1 & 32 \\
\hline Ponta Grossa & 4,5 & 6,2 & 0,3 & 4,3 & 1,2 & 0,6 & 0,12 & 6,22 & 2,4 & 13,3 & 31 \\
\hline Umuarama & 4,1 & 5,8 & 0,7 & 5,8 & 1,2 & 0,4 & 0,13 & 7,53 & 3,1 & 13,3 & 23 \\
\hline
\end{tabular}

Al: alumínio; H+Al: hidrogênio + alumínio; Ca: cálcio; Mg: magnésio; K: potássio; CTC: capacidade de troca de cátions; V: saturação por bases; SMP: método de Shoemaker, Mac Lean e Pratt; *Municípios de coleta do tipo de solo representativo da área correspondente às regionais da Região metropolitana de Curitiba, União da Vitória, Litoral e Arapongas, respectivamente. 
campo foi obtida através da mesa de tensão (EMBRAPA, 1997), visando sua manutenção a 80\% com a adição de água deionizada, nos solos incubados durante o período do experimento.

Para a obtenção das curvas de pH, empregou-se o ensaio de incubação para lodo de esgoto alcalinizado aplicado ao solo, de acordo com a metodologia oficial (BRASIL 2006). Cada experimento seguiu delineamento inteiramente casualizado com 5 tra-

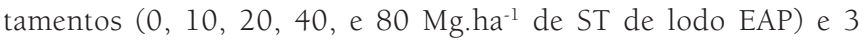
repetições. Para determinação do $\mathrm{pH}$ utilizou-se solução de $\mathrm{CaCl}_{2}$ 0,01 M relação solo/solução (1:2,5) (EMBRAPA, 1997). O efeito do tempo sobre $\mathrm{pH}$ foi avaliado em amostras de solo incubado aos $0,7,14,30,45$ e 60 dias.

Os dados obtidos foram analisados estatisticamente pelo programa R, fazendo-se a análise da variância. Para cada solo, visando identificar a taxa de aplicação máxima anual, a análise de regressão polinomial do $\mathrm{pH}$ versus dose de lodo EAP foi realizada e considerou-se o limite superior do intervalo de predição com 95\% de confiabilidade (DRAPER \& SMITH, 1998), para indicar a quantidade de lodo EAP que não ultrapassasse o pH 7,0, aumentando a segurança em relação ao uso direto da equação. Enquanto a equação representa a média das ocorrências, podendo resultar em valores futuros tanto inferiores quanto superiores ao $\mathrm{pH}$ 7,0, o limite superior do intervalo de predição, com 95\% de confiabilidade, melhor atende a legislação, pois aumenta a probabilidade de não se ultrapassar o pH 7,0 em solos que recebam a quantidade de lodo EAP recomendada por esse limite.

Para a interpretação da relação entre a taxa máxima obtida e os atributos químicos e físicos, os mesmos foram divididos em quatro grupos, baseados na média (M) e no desvio padrão (DP) dos valores obtidos para os 20 solos: Grupo I - taxa muito alta, maior que M+2DP; Grupo II — taxa alta, M+DP; Grupo III — taxa média, M-DP; Grupo IV - taxa baixa, menor que M-2DP.

As correlações entre a TAMA e os atributos do lodo e do solo foram realizadas com auxílio do programa estatístico Assistat (SILVA \& AZEVEDO, 2002) e as correlações múltiplas através de matriz de correlação pelo programa Excel. A equação de estimativa da dose de lodo EAP levando em consideração os atributos do solo que apresentaram correlações significativas foi obtida através de matriz de correlação multivariada, para taxa máxima (Equação 1).

$y=a+b x_{1}+c x_{2}+d x_{3}+e x_{4}+\ldots+i x_{n}$

Onde,

y: dose de lodo EAP;

$\mathrm{x}_{1} \mathrm{ax}_{\mathrm{n}}$ : atributos do solo que apresentaram correlação.

Para avaliar o potencial dos métodos de recomendação de corretivos agrícolas, visando o uso agrícola do lodo EAP, os valores de taxas obtidos pelo método de incubação para atingir diversos
$\mathrm{pH}$ foram comparados com as quantidades calculadas pelo método V\%, conforme descrito por Andreoli, Pegorini e Fernandes (2001) (Equação 2) e pelo pH referência (SMP) conforme a tabela apresentada em SBCS (2004).

Dose de lodo $\mathrm{EAP}=\underline{(\mathrm{V} \% \text { a ser atingido }-\mathrm{V} \% \text { do solo }) * \mathrm{CTC}_{\mathrm{pH} 7}}$

Onde,

$\mathrm{CTC}_{\mathrm{pH} 7}$ : capacidade de troca de cátions em pH 7;

PRNT: poder relativo de neutralização total.

Foram realizadas correlações simples para a comparação entre o método de incubação para os pH 7,0, 6,0 e 5,5 e os métodos de recomendação agrícola de lodo EAP.

\section{Resultados e discussão}

\section{Taxa de aplicação máxima anual de lodo de esgoto higienizado por processo de estabilização alcalina prolongada}

A Tabela 4 apresenta os valores de TAMA para os solos das 20 regionais, bem como os parâmetros estatísticos obtidos. Verifica-se a grande variação nos valores, o que corrobora os resultados obtidos por Serrat et al. (2011), sendo as menores taxas observadas para os solos de Umuarama e Ponta Grossa (10,0 e 10,8 Mg.ha-1) e as maiores para os solos de Francisco Beltrão e Cascavel (>80,0 Mg.ha-1). Para esses dois últimos não foi possível determinar a taxa máxima pela curva, uma vez que o valor do $\mathrm{pH}$ não alcançou 7,0, durante os 60 dias de incubação, chegando no máximo a pH 6,8. Isso indica que a metodologia utilizada não contempla todos os tipos de solos, embora os resultados obtidos nessas localidades permitam a obtenção de informações sob o aspecto agronômico, uma vez que os $\mathrm{pH}$ atingidos estão na faixa adequada para a maioria das plantas cultivadas (MELLO et al., 1984; MALAVOLTA, 2006)

O valor médio obtido para a TAMA, com desvio padrão de 23 , foi de $39 \mathrm{Mg}$.ha-1 de lodo EAP. Para a interpretação das relações entre a TAMA de lodo EAP para os 20 solos e os atributos químicos, físicos e físico-químicos de cada um deles, a Tabela 4, conforme citado na metodologia, apresenta quatro grupos de solos, quais sejam: Grupo I - valores superiores a 62,1 Mg.ha-1; Grupo II - valores

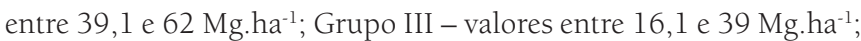
Grupo IV - valores menores que $16 \mathrm{Mg} \cdot \mathrm{ha}^{-1}$.

Os solos do Grupo I que apresentaram as maiores TAMA (das regionais de Francisco Beltrão, Cascavel, Pato Branco e Região Metropolitana de Curitiba) caracterizam-se por serem solos desenvolvidos (Latossolos) e apresentarem altos teores de argila e carbono orgânico. O elevado teor de argila é dado pelo material de origem de 
basalto (Francisco Beltrão, Cascavel e Pato Branco) e argilito (região metropolitana de Curitiba). Todos os solos apresentavam $\mathrm{pH}$ próximo a 4,0, sendo por isso considerado muito ácido em função do grau de intemperismo (FONTES; CAMARGO; SPOSITO, 2001). A combinação da textura argilosa e teor de matéria de orgânica conferem, a estes solos, alto poder tamponante, o que pode ser observado pelos valores elevados de $\mathrm{Al},(\mathrm{H}+\mathrm{Al}), \mathrm{CTC}$, e pelos valores do $\mathrm{pH}_{\text {SMP }}$ (Tabelas 2 e 3 ).

Tabela 4 - Taxa máxima de aplicação anual de lodo de esgoto higienizado por processo de estabilização alcalina prolongada considerando todos os tempos de incubação, para 20 solos paranaenses, correspondente a cada regional atendida pela Companhia de Saneamento do Paraná.

\begin{tabular}{|c|c|c|c|c|}
\hline Regional & Grupo* & $\begin{array}{l}\text { TAMA }^{\#} \\
\text { Mg.ha }^{-1}\end{array}$ & $\mathbf{R}^{2 \dagger}$ & $\begin{array}{l}\text { Erro padrão } \\
\text { residual }^{\ddagger}\end{array}$ \\
\hline Francisco Beltrão & \multirow{4}{*}{ I } & $>80,0$ & 0,964 & 0,1795 \\
\hline Cascavel & & $>80,0$ & 0,972 & 0,1305 \\
\hline $\begin{array}{l}\text { Região Metropolitana de } \\
\text { Curitiba }\end{array}$ & & 74,2 & 0,977 & 0,1523 \\
\hline Pato Branco & & 68,8 & 0,984 & 0,1455 \\
\hline Guarapuava & \multirow{4}{*}{ II } & 59,3 & 0,978 & 0,1645 \\
\hline Toledo & & 52,4 & 0,963 & 0,2213 \\
\hline Apucarana & & 47,1 & 0,940 & 0,2277 \\
\hline Campo Mourão & & 46,5 & 0,983 & 0,1527 \\
\hline União da Vitória & \multirow{8}{*}{ III } & 38,5 & 0,961 & 0,2868 \\
\hline Litoral & & 36,5 & 0,854 & 0,5090 \\
\hline Telêmaco Borba & & 28,6 & 0,989 & 0,1637 \\
\hline Maringá & & 27,6 & 0,987 & 0,1466 \\
\hline Santo Antônio da Platina & & 25,4 & 0,989 & 0,1255 \\
\hline Londrina & & 22,7 & 0,935 & 0,2767 \\
\hline Foz do Iguaçu & & 22,4 & 0,988 & 0,1163 \\
\hline Arapongas & & 21,2 & 0,981 & 0,2346 \\
\hline Cornélio Procópio & \multirow{4}{*}{ IV } & 15,4 & 0,971 & 0,2135 \\
\hline Paranavaí & & 12,3 & 0,953 & 0,3033 \\
\hline Ponta Grossa & & 10,8 & 0.968 & 0.2673 \\
\hline Umuarama & & 10,0 & 0.985 & 0.2112 \\
\hline
\end{tabular}

TAMA: taxa máxima de aplicação anual; *Separação em relação à média $\left(39 \mathrm{~kg} \mathrm{ha} \mathrm{F}^{-1}\right)$ considerando o desvio padrão $=23$; * $y \sim f+a{ }^{*} x^{\wedge} b /\left(c^{\wedge} b+x^{\wedge} b\right)$, onde $x$ : dose de lodo de esgoto higienizado por processo de estabilização alcalino prolongado (lodo EAP) e y: pH; 'Taxa de lodo EAP para atingir pH 7em 60 dias, pelo limite de predição; ‘para 86 graus de liberdade.

Tabela 5 - Correlações simples significativas entre as características dos lodos e dos solos com a taxa de aplicação máxima anual de lodo de esgoto higienizado por processo de estabilização alcalina prolongada.

\begin{tabular}{|c|c|c|c|c|}
\hline & TAMA & Argila & $\mathrm{C}$ & $\mathrm{H}+\mathrm{Al}$ \\
\hline $\mathrm{pH}$ do lodo EAP & $-0,57^{\star *}$ & & & \\
\hline Argila & $0,68^{* *}$ & & & \\
\hline $\mathrm{C}$ & $0,56 * \star$ & $0,55^{\star}$ & & \\
\hline $\mathrm{H}+\mathrm{Al}$ & 0,70 ** & - & $0,51^{\star}$ & \\
\hline CTC pH 7,0 & 0,80 ** & $0,53^{*}$ & 0,64 ** & $0,82^{* *}$ \\
\hline
\end{tabular}

Os solos do Grupo II têm como material de origem o basalto e apresentam a mesma característica que o Grupo I, como elevado teores de C e textura argilosa, mas com menores valores de TAMA. Isso se deve possivelmente às variações entre as atividades das argilas e/ou a outros fatores (MOTTA \& LIMA, 2006), com destaque para o PN (54\%) do lodo utilizado no solo de Guarapuava e para o $\mathrm{pH}_{\mathrm{CaCl}_{2}}$ de 4,9 e V de $56 \%$ do solo de Apucarana (Tabela 3). Se essa subdivisão de grupos fosse

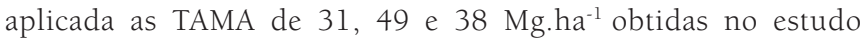
de Marin et al. (2010) para Cambissolo Háplico, Cambissolo Húmico e Latossolo Bruno, todos com características distróficas, verifica-se que apenas o Cambissolo Húmico utilizado seria pertencente a este grupo, pois apesar do alto teor de C, apresentava menores teores de argila que os demais solos.

Os solos do Grupo III apresentaram características variadas, sendo os das regionais de Maringá e Foz do Iguaçu aqueles que possuíam elevados teores de argila e carbono, porém com valores elevados de V\% (51 e 62\%, respectivamente). Os solos das regionais de União da Vitória (Rio Negro), do litoral (Matinhos) e de Telêmaco Borba, embora tivessem baixos teores de argila, apresentaram as taxas mais elevadas dentro do grupo III devido, possivelmente, ao baixo V\% (Tabela 4).

Dos solos do Grupo IV, que apresentam as menores TAMA, os solos de Paranavaí, Ponta Grossa e Umuarama contêm baixos teores de argila e carbono, com pH que variaram de muito baixo a baixo (SERRAT; KRIEGER; MOTTA, 2006), tendo como material de origem arenitos. Para estes três solos, a combinação de textura arenosa e baixo teor de $\mathrm{C}$ determina um baixo poder tamponante, o que é evidenciado no solo de Paranavaí pelo seu elevado $\mathrm{pH}_{\mathrm{SMP}}(6,9)$. O solo de Cornélio Procópio apresentou baixa resposta a adição de material corretivo por apresentar características eutróficas, e mesmo tendo sido coletado em área nativa, apresentou saturação por bases de $53 \%$ (Tabela 3).

A Tabela 5 apresenta apenas as correlações que foram significativas entre as características dos lodos e dos solos com a TAMA de lodo EAP. Dentre os atributos dos solos, os que apresentaram as melhores correlações foram a $\mathrm{CTC}_{\mathrm{pH} 7,0}$ e a acidez potencial $(\mathrm{H}+\mathrm{Al})$, ambos resultam da associação de atributos como pH do solo, o teor e tipo de argila e teor de matéria orgânica (ERNANI, 2008). Depois, seguem o teor de argila e de carbono, indicando também a contribuição direta desses atributos. Como a correlação foi significativa apenas para o pH do lodo após a higienização, não ocorrendo o mesmo para o PN, sólidos totais e pH do lodo bruto, verifica-se que as características do solo foram mais determinantes para a recomendação desse tipo de resíduo.

A partir dos atributos dos solos citados na Tabela 5, em uma análise de correlação multivariada, determinou-se parâmetros numéricos para resolução da Equação 1, obtendo-se a Equação 3. 
$y=-13,44457-0,67401 x_{1}+4,61244 x_{2}+0,45389 x_{3}-0,08944 x_{4}$

Onde,

y: dose de lodo EAP (Mg.ha-1 ${ }^{-1}$;

$\mathrm{x}_{1}: \mathrm{CTC}_{\mathrm{pH} 7}\left(\mathrm{cmol}_{\mathrm{c}} \mathrm{dm}^{-3}\right)$;

$\mathrm{x}_{2}: \mathrm{H}+\mathrm{Al}\left(\mathrm{cmol}_{\mathrm{c}} \mathrm{dm}^{-3}\right)$;

$\mathrm{x}_{3}$ : $\operatorname{argila}\left(\mathrm{g} \cdot \mathrm{kg}^{-1}\right)$;

$\mathrm{x}_{4}$ : carbono orgânico $\left(\mathrm{g} \cdot \mathrm{dm}^{-3}\right)$.

Os valores calculados a partir dessa equação e dos resultados das Tabelas 2 e 3 foram correlacioandos com os valores das taxas obtidos pelo método da incubação (Tabela 4), conforme a Figura 1.

A boa correlação obtida permite o uso desta equação na estimativa da TAMA de lodo EAP (y) a partir de resultados de análises de rotina utilizadas na avaliação da fertilidade do solo.

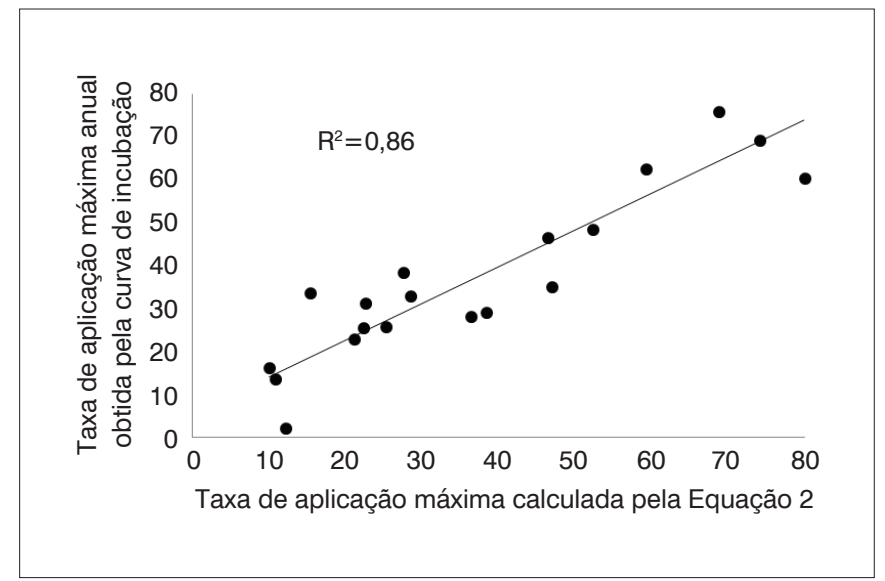

Figura 1 - Correlação entre as taxas de aplicação máxima anual de lodo de esgoto higienizado por processo de estabilização alcalina prolongada obtidas pelas curvas de incubação para pH 7,0 de 20 solos do estado do Paraná e os valores calculados pela Equação 2: $y=-13,445$ $0,674\left(\mathrm{CTC}_{\mathrm{pH} 7}\right)+4,612(\mathrm{H}+\mathrm{Al})+0,454($ argila $)-0,089(\mathrm{C})$.

\section{Comparação entre métodos de recomendação agrícola de lodo de esgoto higienizado por processo de estabilização alcalina prolongada}

A Tabela 6 apresenta as correlações simples entre os métodos avaliados (saturação por bases e pH referência) com os resultados dos três pH $(7,0,6,0$ e 5,5) obtidos pelo método da incubação, utilizado como padrão. Observa-se que para todos os casos o valor do índice $r$ foi superior a 0,73, indicando alta correlação para todos os pH estudados.

Além, da correlação entre os métodos, para a recomendação agronômica é necessário observar aspectos da legislação (BRASIL, 2006) e também os relacionados às culturas (MALAVOLTA, 2006 SBCS, 2004).

As doses de lodo EAP obtidas para pH em $\mathrm{CaCl}_{2}$ 7,0, 6,0 e 5,5, utilizando o método de incubação (BRASIL, 2006; PARANÁ, 2009), bem como os métodos de saturação por bases (V\%) e pH referência (SMP) são apresentadas na Tabela 7

$\mathrm{Na}$ quarta coluna de dados desta tabela encontram-se as doses de lodo EAP calculadas considerando a cal com PN=124\% ( $1 / 3$ deste valor para a adição de cal a $50 \%$ dos sólidos totais) para uma cultura que necessite $\mathrm{V} \%=70$, conforme Andreoli, Pegorini e Fernandes. (2001). Observa-se que esses valores não atingiriam $\mathrm{pH} 7,0$, pelo limite superior do intervalo de predição das curvas de incubação (coluna 1), ficando inclusive, muito abaixo do valor estimado para este $\mathrm{pH}$. Ao comparar-se com os pH 6,0 e 5,5, também da curva de incubação (colunas 2 e 3), essa diferença diminui. Porém a quantidade de lodo EAP recomendada pelo método da saturação por bases (PN da cal), para maioria dos solos, continua sendo inferior aos valores obtidos pelo método da incubação (Tabela 7).

No caso das doses calculadas a partir deste mesmo método (V\%), mas utilizando-se o PN de cada lodo EAP (coluna 5), os

Tabela 6 - Correlações simples entre as taxas de aplicação máxima anual de lodo de esgoto higienizado por processo de estabilização alcalina prolongada obtidas pelas curvas de incubação para pH 7,0, 6,0 e 5,5 e as doses calculadas pelo método da saturação por bases e pelo pH referência.

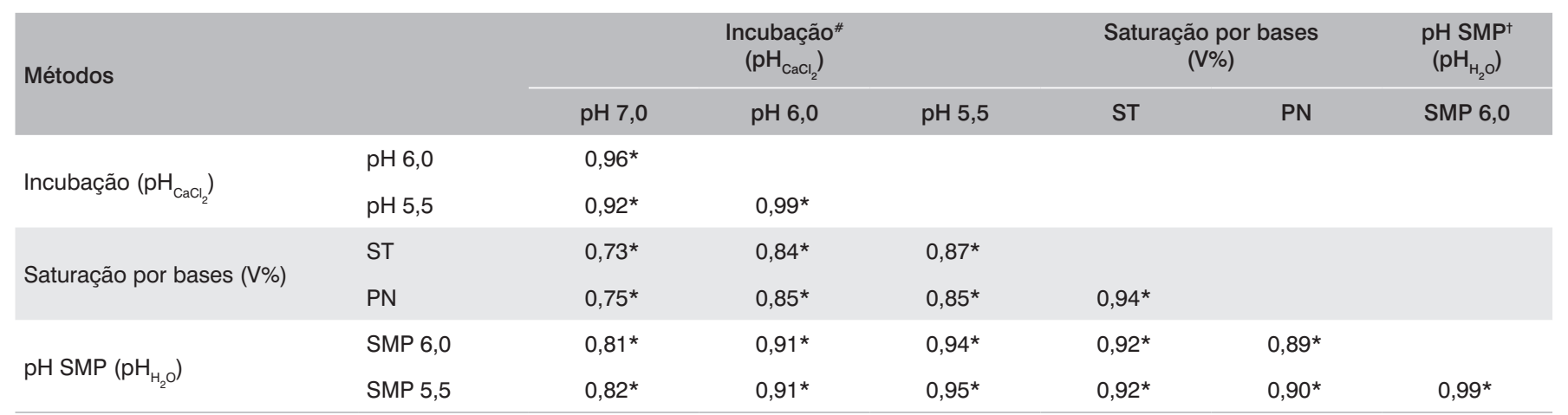

ST: sólidos totais; PN: poder de neutralização; pH SMP: pH referência (SBCS, 2004); \#curva de incubação obtida no limite superior do intervalo de predição a 95\%; ${ }^{\mathrm{c}}$ calculado para atingir V\% de 70\% (ANDREOLI; PEGORINI; FERNANDES, 2001); * coeficientes de correlação significativos a 1\%. 
Tabela 7 - Doses de lodo de esgoto higienizado por processo de estabilização alcalina prolongada recomendadas a partir das curvas de incubação para $\mathrm{pH}$ 7,0, 6,0 e 5,5 e pelos métodos de saturação por bases e pH referência.

\begin{tabular}{|c|c|c|c|c|c|c|c|}
\hline \multirow{5}{*}{ Regional } & \multicolumn{7}{|c|}{ Métodos de recomendação } \\
\hline & \multicolumn{3}{|c|}{ Curva de incubação* } & \multicolumn{2}{|c|}{ Saturação por bases** } & \multirow{2}{*}{\multicolumn{2}{|c|}{$\frac{\mathrm{pH} \text { SMP }}{\mathrm{pH}_{\mathrm{H}_{2} \mathrm{O}}}$}} \\
\hline & \multicolumn{3}{|c|}{$\mathrm{pH}_{\mathrm{CaCl}_{2}}$} & \multirow{2}{*}{$\mathrm{PN}$ da cal ${ }^{\dagger}$} & \multirow{2}{*}{ PN do lodo $\#$} & & \\
\hline & 7,0 & 6,0 & 5,5 & & & 6,0 & 5,5 \\
\hline & \multicolumn{7}{|c|}{$\left(M_{g} \cdot h^{-1}\right)$} \\
\hline Francisco Beltrão & $>80$ & 60,2 & 47,7 & 26,5 & 27,1 & 63,0 & 45,0 \\
\hline Cascavel & $>80$ & 49,4 & 33,1 & 14,0 & 12,6 & 29,7 & 19,8 \\
\hline Região Metropolitana de Curitiba & 74,2 & 48,9 & 33,8 & 23,4 & 32,8 & 39,9 & 28,8 \\
\hline Pato Branco & 68,8 & 43,6 & 30,3 & 23,3 & 31,1 & 45,3 & 32,7 \\
\hline Guarapuava & 59,3 & 30,6 & 23,9 & 18,0 & 13,8 & 32,1 & 23,1 \\
\hline Toledo & 52,4 & 27,1 & 19,1 & 11,5 & 12,1 & 20,4 & 12,6 \\
\hline Apucarana & 47,1 & 19,9 & 10,0 & 4,2 & 5,2 & 11,1 & 6,0 \\
\hline Campo Mourão & 46,5 & 24,8 & 16,6 & 10,4 & 9,3 & 18,3 & 11,1 \\
\hline União da Vitória & 38,5 & 27,7 & 17,8 & 22,8 & 19,9 & 24,9 & 15,9 \\
\hline Litoral & 36,5 & 22,0 & 14,6 & 12,9 & 10,3 & 20,4 & 12,6 \\
\hline Telêmaco Borba & 28,6 & 20,2 & 15,3 & 15,9 & 14,6 & 27,3 & 18,0 \\
\hline Maringá & 27,6 & 16,3 & 11,0 & 6,2 & 7,5 & 16,2 & 9,6 \\
\hline Santo Antônio da Platina & 25,4 & 11,9 & 6,7 & 7,1 & 6,6 & 14,4 & 8,4 \\
\hline Londrina & 22,7 & 9,4 & 4,2 & 4,0 & 4,3 & 5,4 & 2,4 \\
\hline Foz do Iguaçu & 22,4 & 9,2 & 4,4 & 2,2 & 1,9 & 6,6 & 3,0 \\
\hline Arapongas & 21,2 & 17,7 & 13,6 & 10,8 & 10,8 & 18,3 & 11,1 \\
\hline Cornélio Procópio & 15,4 & 10,1 & 7,7 & 9,1 & 6,5 & 20,4 & 12,6 \\
\hline Paranavaí & 12,3 & 7,9 & 5,3 & 3,4 & 3,3 & 2,0 & 0,47 \\
\hline Ponta Grossa & 10,8 & 7,1 & 4,8 & 5,9 & 4,1 & 6,6 & 3,0 \\
\hline Umuarama & 10,0 & 8,7 & 6,8 & 8,6 & 9,0 & 12,6 & 6,9 \\
\hline
\end{tabular}

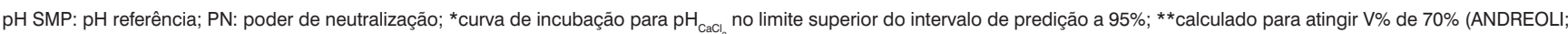
PEGORINI; FERNANDES, 2001); †PN 124\%; " $P N$ determinado para cada lodo após a alcalinização.

valores foram muito próximos aos obtidos na coluna 4. Como na operação de higienização em escala real, a quantidade de cal adicionada nem sempre está na proporção exata de um terço dos sólidos totais do lodo, como nesse trabalho, a consequência para os diferentes lotes, será a de maior variação do poder neutralizante (SERRAT et al., 2011). Assim, o conhecimento do PN de cada lote de lodo EAP, pode contribuir para melhor adequação das doses recomendadas para fins agrícolas.

Observa-se na coluna 6 da Tabela 6 , que as doses recomendas pelo método do $\mathrm{pH}$ SMP (SBCS, 2004) para atingir $\mathrm{pH}_{\mathrm{H}_{2} \mathrm{O}}$ 6,0 apresentaram limitações para os solos de Cornélio Procópio e Umuarama, os quais atingiram valores superiores ao $\mathrm{pH}_{\mathrm{CaCl}_{2}}$ 7,0 (coluna 1). Contudo, as doses recomendadas por esse mesmo método, para atingir $\mathrm{pH}_{\mathrm{H}_{2} \mathrm{O}} 5,5$ (coluna 7 ), resultaram em valores seguros, ou seja, que não ultrapassam o pH 7 (BITTENCOURT et al., 2010) e foram próximos aos valores obtidos pelo método da incubação para o $\mathrm{pH}_{\mathrm{CaCl}_{2}}$ 5,5 (coluna 3).

Desta forma, é possível indicar os métodos de saturação por bases $(\mathrm{V} \%)$ e $\mathrm{pH}$ de referência para $\mathrm{pH}_{\mathrm{H}_{2} \mathrm{O}}$ 5,5 como métodos de recomendação de doses de lodo EAP para utilização agrícola, pois para os 20 solos, as doses recomendadas não atingiram a dose máxima estabelecida pelo critério do pH. Ressalta-se ainda que esta dose recomendada pelo projeto agronômico também depende de outros critérios da legislação, como por exemplo, o critério do nitrogênio o qual limita a dose conforme a sua concentração (BRASIL, 2006).

\section{Conclusão}

Em função do critério de elevação de pH, conforme estabelece a legislação vigente, e considerando as características do lote de lodo de esgoto EAP utilizado (higienizado a 50\% dos ST) e do solo selecionado para cada regional, os resultados indicam que as taxas de aplicação máxima anual de lodo EAP, variaram de $10 \mathrm{Mg} \cdot \mathrm{ha}^{-1}$ a $>80 \mathrm{Mg} \cdot \mathrm{ha}^{-1}$ para as 20 regionais estudadas; os atributos químicos (CTC, $\mathrm{H}+\mathrm{Al}$ e carbono) e físicos (argila) que conferem resistência à elevação de pH são os que melhor definem a determinação da taxa de aplicação máxima anual, devido à ocorrência de correlação 
positiva, ou seja, quanto maiores os valores desses atributos no solo maior será a quantidade de lodo EAP a ser aplicada; os métodos do $\mathrm{V} \%$ e do $\mathrm{pH}$ referência (SMP) para $\mathrm{pH}_{\mathrm{H}_{2} \mathrm{O}}$ 5,5 mostraram-se seguros quanto à recomendação agrícola.

\section{Agradecimento}

Ao Professor Jorge Luiz Moretti de Souza, do Departamento de Solos e Engenharia Agrícola da UFPR, pelo auxílio nas análises estatísticas.

\section{Referências}

ANDREOLI, C.V.; PEGORINI, E.S.; FERNANDES, F. (2001) Disposição do lodo no solo. In: ANDREOLI, C.V.; SPERLING, M. VON; FERNANDES, F. Lodo de esgotos: tratamento e disposição final. v. 1, Belo Horizonte, UFMG, Departamento de Engenharia Sanitária e Ambiental, SANEPAR, p. 319-398.

BHERING, S.B.; SANTOS, H.G. (2008) Mapa de solos de estado do Paraná: legenda atualizada. Rio de Janeiro, EMBRAPA Floresta, EMBRAPA Solos, Instituto Agronômico do Paraná, 74 p.

BITTENCOURT, S.; ANDREOLI, C.V.; MOCHIDA, G.A; SOUZA, L.M.K.M.; SERRAT, B.M. (2009) Aspectos agronômicos do uso agrícola de lodo de esgoto - região metropolitana de Curitiba. Revista AIDIS de Ingeniería y Ciencias Ambientales, v. 2, n. 1, p. 1-11.

BITTENCOURT, S.; ANDREOLI, C.V.; MOCHIDA, G.A; SOUZA, L.M.K.M.; SERRAT, B.M. (2010) Uso agrícola de lodo de esgoto no estado do Paraná. In: COSCIONE, A.R.; NOGUEIRA, T.A.; PIRES, A.M.M. (ed.). Uso agrícola de lodo de esgoto, aspectos agronômicos do uso agrícola de lodo de esgoto. Botucatu, FEPAF, p. 281-297.

BRASIL. Ministério do Meio Ambiente. Conselho Nacional do Meio Ambiente. (2006) Resolução CONAMA no 375 de 29 de agosto de 2006. Define critérios e procedimentos, para o uso agrícola de lodos de esgoto gerados em estações de tratamento de esgoto sanitário. Diário Oficial da União de 30 de agosto de 2006. Disponível em: <http://www.mma. gov.br/port/conama/res/res06/res37506.pdf > . Acesso em: fev. 2013.

CHUEIRI, W.A.; SERRAT, B.M.; BIELE, J.; FAVARETTO, N. (2007). Lodo de esgoto e fertilizante mineral sobre parâmetros do solo e de plantas de trigo. Revista Brasileira Engenharia Agrícola Ambiental, v. 11, n. 5, p. 502-508.

CORRÊA, R.S.; WHITE, R.E.; WEATHERLEY, A.J (2005) Biosolids effectiveness to yield ryegrass based on their nitrogen content. Scientia Agricola, v. 62, n. 3, p. 274-280

DRAPER, N.R.; SMITH JR., H. (1998) Apllied regression analysis. New York, John Wiley, $706 \mathrm{p}$.

EMPRESA BRASILEIRA DE PESQUISA AGROPECUÁRIA (EMBRAPA). Serviço Nacional de Levantamento e Conservação do Solo. (1997) Manual de métodos de análise do solo. Rio de Janeiro, EMBRAPA, 212 p.

ERNANI, P.R. (2008) Química do solo e disponibilidade de nutrientes. Lages, UDESC, $230 \mathrm{p}$.

FIA, R.; MATOS, A.T.; AGUIRRE, C.I. (2005) Características químicas de solo adubado com doses crescentes de lodo de esgoto caleado. Engenharia na Agricultura, v. 13, n. 4, p. 287-299.
FONTES, M.P.F.; CAMARGO, O.A.; SPOSITO, G. (2001) Eletroquímica das partículas coloidais e sua relação com a mineralogia de solos altamente intemperizados. Scientia Agricola, v. 58, n. 3, p. 627-646.

MALAVOLTA, E. (2006) Manual de nutrição mineral de plantas. São Paulo, Agronômica Ceres, 638 p.

MARIN, L.M.K.S.; BITTENCOURT, S.; ANDREOLI, C.V.; CARAFINI, C.; LIMA, M.R.; SERRAT, B.M.; MOCHIDA, G.A. (2010) Determinação da taxa de aplicação máxima anual de lodo de esgoto higienizado por processo alcalino em solos da região metropolitana de Curitiba. Engenharia Sanitária e Ambiental, v. 15, n. 2, p.113-118.

MARQUES, R. \& MOTTA, A.C.V. (2003) Análise química do solo para fins de fertilidade. In: LIMA, R.M. (org.). Manual de diagnóstico da fertilidade e manejo dos solos agrícola. Curitiba, UFPR/DSEA, p. 81-102.

MELLO, F.A.F.; BRASIL SOBRINHO, M.O.C.; ARZOLLA, S.; SILVEIRA, R.J.; COBRA NETTO, A; KIEHL, J.C. (1984) Fertilidade do solo. 2 ed. São Paulo, Nobel, 400 p.

MOTTA, A.C.V. \& LIMA, M.R. Princípios de calagem. (2006). In: LIMA, M.R. (ed.). Diagnóstico e recomendações de manejo do solo: aspectos teóricos e metodológicos. Curitiba, UFPR, p. 191-232.

NOLLA, A. \& ANGHINONI, I. (2004) Métodos utilizados para a correção da acidez do solo no Brasil. Revista Ciências Exatas e Naturais, v. 6, n. 1, p. $97-111$

PARANÁ (estado). Secretaria de Estado de Meio Ambiente e Recursos Hídricos. (2009) Resolução SEMA nº 021 de 22 de abril de 2009. Dispõe sobre licenciamento ambiental, estabelece condições e padrões ambientais e dá outras providências, para empreendimentos de saneamento. Diário Oficial do Estado do Paraná, Curitiba, n. 7.962, p. 13-16.

PIKE, E.B. \& DAVIS, R.D. (1984) Stabilisation and disinfection: their relevance to agricultural utilization of sludge. Stabilisation and Disinfections of Sewage Sludge, v. 3, p. 1-29.

SERRAT, B.M. \& KRIEGER, K.I.; MOTTA, A.C.V. (2006) Considerações sobre interpretação de análise de solos (com exemplos). In: LIMA, M. R. (ed.). Diagnóstico e recomendações de manejo do solo: aspectos teóricos e metodológicos. Curitiba, UFPR, p. 125-142.

SERRAT, B.M.; SANTIAGO, T.R.; BITTENCOURT, S.; MOTTA, A.C.V.; SILVA, L.A.T.P. \& ANDREOLI, C.V. (2011) Taxa de aplicação máxima anual de lodo de esgoto higienizado pelo processo de estabilização alcalina: estudo comparativo de curvas de $\mathrm{pH}$ de solos. Revista Brasileira de Ciências Ambientais, n. 19, p. 30-37. 
SILVA, F.A.S. \& AZEVEDO, C.A.V. (2002) Versão do programa computacional Assistat para o sistema operacional Windows. Revista Brasileira de Produtos Agroindustriais, v. 4, n. 1, p. 71-78.

SOCIEDADE BRASILEIRA DE CIÊNCIA DO SOLO (SBCS). Comissão de Química e Fertilidade do Solo. (2004) Manual de adubação e calagem para os Estados do Rio Grande do Sul e Santa Catarina. 10ª ed. Porto Alegre, SBCS, Núcleo Regional Sul, 400 p.

TEDESCO, M.J.; GIANELLO, C.; BISSANI, C.A.; BOHNEN, H.; VOLKWEISS, S.J. (1995) Análise de solo, plantas e outros materiais. 2 ed. Boletim Técnico, 5. Porto Alegre, UFRGS, 174 p. 